\title{
Magnetic Monopoles, Center Vortices and Topology of Gauge Fields
}

\author{
H. Reinhardt*, M. Engelhardt, K. Langfeld, M. Quandt, A. Schäfke, a \\ anstitut für Theoretische Physik, Universität Tübingen, Auf der Morgenstelle 14, D-72076 Tübingen, \\ Germany
}

The topological properties of magnetic monopoles and center vortices arising, respectively, in Abelian and center gauges are studied in continuum Yang-Mills Theory. For this purpose the continuum analog of the maximum center gauge is constructed.

\section{Introduction}

Recent lattice calculations have given strong evidence for two confinement scenarios: 1 . the dual Meissner effect [1], which is based on a condensate of magnetic monopoles in the QCD vacuum and 2. the center vortex picture [2], where the vacuum consists of a condensate of magnetic flux tubes which are closed due to the Bianchi identity. There are also lattice calculations which indicate that the spontaneous breaking of chiral symmetry, which is related to the topology of gauge fields, is caused by these objects, i.e. by either magnetic monopoles [3] or center vortices [1]. In this talk we would like to discuss the topological properties of magnetic monopoles and center vortices. We will first show that in Polyakov gauge the magnetic monopoles completely account for the non-trivial topology of the gauge fields. Subsequently, we will extend the notion of center vortices to the continuum. We will present the continuum analog of the maximum center gauge fixing and the Pontryagin index of center vortices.

\section{Magnetic monopoles and topology}

The magnetic monopoles arise in Yang-MillsTheories in the so called Abelian gauges [5]. For the study of these monopoles in the continuum theory the Polyakov gauge is particularly convenient. In this gauge one diagonalizes the Polyakov

\footnotetext{
*talk presented by H. Reinhardt at Lattice'99, Pisa, Italy.
}

loop

$\Omega(\vec{x})=P e^{-\int_{0}^{T} d x_{0} A_{0}\left(x_{0}, \vec{x}\right)}=V^{+} \omega V$

which fixes $V \in S U(N) / U(1)^{N-1}$ i.e. the coset part of the gauge group. Magnetic monopoles arise as defects of the gauge fixing which occur when at isolated points in space $\vec{x}_{i}$ the Polyakov loop becomes a center element

$\Omega\left(\vec{x}_{i}\right)=(-1)^{n_{i}}, \quad n_{i}$ : integer

The field $A^{V}=V A V^{+}+V \partial V^{+}$develops then magnetic monopoles. These monopoles have topologically quantized magnetic charge [6] given by the winding number

$m[V] \in \Pi_{2}(S U(2) / U(1))$

of the mapping $V(\vec{x})$ from a sphere $S_{2}$ around the magnetic monopole into the coset $S U(2) / U(1)$ of the gauge group.

In the Polyakov gauge the Pontryagin index can be exactly expressed in terms of magnetic charges. If we assume a compact space-time manifold and that there are only point-like defects of gauge fixing, i.e magnetic monopoles are the only magnetically charged objects arising after gauge fixing, the Pontryagin index is given by 6]

$\nu=\Sigma_{i} n_{i} m_{i}$

The summation runs here over all magnetic monopoles with $m_{i}$ being the magnetic charge of the monopole defined by equation (3) and the integer $n_{i}$ is defined by the value of the Polyakovloop at the monopole position (2). This relation shows that the magnetic monopoles completely account for the non-trivial topology of 
gauge fields, at least in the Polyakov gauge. Unfortunately, in other Abelian gauges like maximum Abelian gauge, such a simple relation between Pontryagin index and topological charges is not yet known. However, in the maximum Abelian gauge correlations between instantons and monopoles has been found, in both analytical and lattice studies [3].

\section{Center vortices in the continuum}

In order to extend the notion of center vortices to the continuum theory let us, undertake a detour through the lattice by putting a given smooth gauge field $A_{\mu}(x)$ on a fine lattice in the standard fashion by introducing the link variables $U_{\mu}(x)=\exp \left(-a A_{\mu}(x)\right)$. For initially smooth gauge fields $A_{\mu}(x)$ and for sufficiently fine lattices all link variables will be close to unity, which represents the "north pole" of the SU(2) group manifold $S_{3}$. However, in the process of gauge fixing, gauge transformations are performed which transform some of the links from the northern to the southern hemisphere. In fact, we can separate each gauge transformation into a transformation which merely switches a link from the northern to the southern hemisphere (or vice versa) and one which rotates link variables inside either the northern or the southern hemisphere but does not switch hemispheres.

In the maximum center gauge condition

$\sum_{x, \mu}\left(T_{r} U_{\mu}(x)\right)^{2} \rightarrow \max$,

which is obviously insensitive to center gauge transformations, one exploits gauge transformations to rotate a link variable as close as possible to a center element. Once the maximum center gauge has been implemented, center projection implies to replace all links by their closest center element. One obtains then a $Z(2)$ lattice which contains $D-1$ dimensional hypersurfaces $\Sigma$ on which all links take a non-trivial center element that is $U=-1$ in the case of $S U(2)$. The $D-2$ dimensional boundaries $\partial \Sigma$ of the hypersurfaces $\Sigma$ represent the center vortices, which, when nontrivially linked to a Wilson loop, yield a center element for the latter.
A carefully analysis shows that the continuum analogies of the center vortices are defined by the gauge potential [7],

$\mathcal{A}_{\mu}(x, \Sigma)=E \int_{\Sigma} d^{D-1} \tilde{\sigma}_{\mu} \delta^{D}(x-\bar{x}(\sigma))$

where $d^{D-1} \tilde{\sigma}_{\mu}$ is the dual of the $D-1$ dimensional volume element. Furthermore, the quantity $E=E_{a} H_{a}$ with $H_{a}$ being the generators of the Cartan algebra represents (up to a factor of $2 \pi$ ) the so called co-weights which satisfy $\exp (-E)=Z \in Z(N)$. Due to this fact the Wilson-loop calculated from the gauge potential (6) becomes,

$W[\mathcal{A}](C)=\exp \left(-\oint_{C} \mathcal{A}\right)=Z^{I(C, \Sigma)}$

where $I(C, \Sigma)$ is the intersection number between the Wilson-loop $C$ and the hypersurface $\Sigma$. The representation, (6), is referred to as ideal center vortex. One should emphasize that the hypersurface $\Sigma$ can be arbitrarily deformed by a center gauge transformation keeping, however, its boundary $\partial \Sigma$, i.e. the position of the center vortex, fixed. Thus for fixed $\partial \Sigma$ the dependence of the gauge potential (6) on the hypersurface itself is a gauge artifact.

The dependence on the hypersurface $\Sigma$ can be removed by performing the gauge transformation

$\varphi(x, \Sigma)=\exp (-E \Omega(x, \Sigma))$

where $\Omega(x, \Sigma)$ is the solid angle subtended by the hypersurface $\Sigma$ as seen from the point $x$. One finds then

$\mathcal{A}_{\mu}(x, \partial \Sigma)=\varphi(x, \Sigma) \partial_{\mu} \varphi^{+}(x, \Sigma)+a_{\mu}(x, \partial \Sigma)$

where

$a_{\mu}(x, \partial \Sigma)=E \int_{\partial \Sigma} d^{D-2} \tilde{\sigma}_{\mu \nu} \partial_{\nu} D(x-\bar{x}(\sigma))$

depends only on the vortex position $\partial \Sigma$ and is referred to as "thin vortex". Here $D(x-\bar{x}(\sigma))$ represents the Green function of the $D$ dimensional Laplacian. In fact, one can show [7] that the thin vortex represents the transversal part of the ideal vortex $a_{\mu}(x, \partial \Sigma)=P_{\mu \nu} \mathcal{A}_{\nu}(x, \Sigma)$ where $P_{\mu \nu}=\delta_{\mu \nu}-\frac{\partial_{\mu} \partial_{\nu}}{\partial^{2}}$ is the usual transversal projector. A careful and lengthy analysis [7] yields then 
the conclusion that the continuum analog of the maximum center gauge fixing is defined by

$\min _{\partial \Sigma} \min _{g} \int \operatorname{Tr}\left(A^{g}-a(\partial \Sigma)\right)^{2}$

where the minimization is performed with respect to all (continuum) gauge transformations $g \in S U(2) / Z(2)$ (which represents per se coset gauge transformations) and with respect to all vortex surfaces $\partial \Sigma$. For fixed thin center vortex field configuration $a(\partial \Sigma)$ minimization with respect to the continuum gauge transformation $\mathrm{g}$ yields the background gauge condition

$\left[\partial_{\mu}+a_{\mu}(\partial \Sigma), A_{\mu}\right]=0$

where the thin vortex field $a_{\mu}(x, \partial \Sigma)$ figures as background gauge field. One should emphasize, however, that the background field has to be dynamically determined for each given gauge field $A_{\mu}(x)$ and thus depends on the latter.

Obviously in the absence of a vortex structure in a considered gauge field $A_{\mu}(x)$ the background gauge condition reduces to the Lorentz gauge $\partial_{\mu} A_{\mu}=0$.

\section{Topology of Center vortices}

Once the center vortex configurations in the continuum are at our disposal, it is straightforward to calculate their Pontryagin index. In the continuum formulation where center vortices live in the Abelian subgroup by construction the direction of the magnetic flux of the vortices is fully kept. The explicit calculation [7] shows that the Pontryagin index $\nu$ of the center vortices is given by $\nu=\frac{1}{4} I(\partial \Sigma, \partial \Sigma)$ where $I(\partial \Sigma, \partial \Sigma)$ represents the self-intersection number of the closed vortex sheet $\partial \Sigma$. A careful analysis shows that for closed orientable surfaces the self intersection number vanishes. In order to have a non-trivial Pontryagin index the vortex surfaces have to be not globally orientable, i.e., they have to consist of orientable pieces. One can further show that at the border between orientable vortex patches magnetic monopole currents flow. It is these monopole currents which make the vortex sheet non orientable since they change the orientation of the magnetic flux. Thus we obtain that even for the center vortices the non-trivial topology is generated by magnetic monopole currents flowing on the vortex sheets. This is consistent with our finding in the Polyakov gauge where the Pontryagin index was exclusively expressed in terms of magnetic monopoles [6].

By implementing the maximum center gauge condition in the continuum one can derive, in an approximate fashion, an effective vortex theory, where the vortex action can be calculated in a gradient expansion. The leading order gives the Nambu-Goto action while in higher orders curvature terms appear. A model based on such an effective vortex action, in fact, reproduces the gross features of the center vortex picture found in numerical lattice simulations.

\section{Acknowledgment:}

This work was supported in part by the DFGRe 856/4-1 and DFG-En 415/1-1.

\section{REFERENCES}

1. G .S. Bali, C. Schlichter and K. Schilling, Prog. Theor. Phys. Suppl. 131 (1998) 645 (1998).

2. L. Del Debbio, M. Faber, J. Greensite and S. Olejnik, Phys. Rev. D55 (1997) 2298;

K. Langfeld, H. Reinhardt and O. Tennert, Phys. Lett. B419 (1998) 317;

K. Langfeld, O. Tennert, M. Engelhardt and H. Reinhardt, Phys. Lett. B452 (1999) 301;

3. S. Thurner, M. Feurstein, H. Markum and W. Sakuler, Phys. Rev. D54 (1996) 3457;

H. Suganuma, S. Sasaki, H. Ichie, F. Araki and O. Miyamura, Nucl. Phys. Proc. Suppl. 53 (1997) 528;

S. Sasaki and O. Miyamura, Phys. Rev. D59 (1999) 094507.

4. Ph. de Forcrand, M. D'Elia, Phys. Rev. Lett. 82 (1999) 4582.

5. G 't Hooft, Nucl. Phys. B190 (1981) 455

6. H. Reinhardt, Nucl. Phys. B503 (1997) 505; M. Quandt, H. Reinhardt and A. Schafke, Phys. Lett. B446 (1999) 290.

7. M. Engelhardt, H. Reinhardt, hep-th/9907139 